\title{
Translation domains in multiferroics
}

\author{
D. Meier ${ }^{\mathrm{a}}$, N. Leo ${ }^{\mathrm{a}}$, T. Jungk ${ }^{\mathrm{b}}$, E. Soergel ${ }^{\mathrm{b}}$, P. Becker ${ }^{\mathrm{c}}$, L. Bohatýc $^{\mathrm{c}}$ and M. Fiebig ${ }^{\mathrm{a} *}$ \\ ${ }^{\mathrm{a}}$ HISKP, Universität Bonn, 53115 Bonn, Germany; ${ }^{\mathrm{b}}$ PI, Universität Bonn, 53115 Bonn, \\ Germany; ${ }^{\mathrm{c} I n s t i t u t ~ f u ̈ r ~ K r i s t a l l o g r a p h i e, ~ U n i v e r s i t a ̈ t ~ z u ~ K o ̈ l n, ~} 50937$ Köln, Germany
}

\begin{abstract}
Translation domains differing in the phase but not in the orientation of the corresponding order parameter are resolved in two types of multiferroics. Hexagonal (h-) $\mathrm{YMnO}_{3}$ is a split-order-parameter multiferroic in which commensurate ferroelectric translation domains are resolved by piezoresponse force microscopy whereas $\mathrm{MnWO}_{4}$ is a joint-order-parameter multiferroic in which incommensurate magnetic translation domains are observed by optical second harmonic generation. The pronounced manifestation of the generally rather "hidden" translation domains in these multiferroics and the associated drastic reduction of symmetry emphasize that the presence of translation domains must not be neglected when discussing the physical properties and functionalities of multiferroics.
\end{abstract}

\section{INTRODUCTION}

During the last decade an abundance of studies on systems with coexisting magnetic and electric order, the so-called multiferroics, disclosed a variety of new and intriguing phenomena such as "unconventional" ferroelectricity or "gigantic" coupling effects between magnetic and electric properties $, 1,2$ The multifarious physics and the technological potential of the multiferroics released a hunt for further members of this material family so that a steadily increasing number of multiferroic compounds is now at our disposal. Irrespective of the diversity of multiferroics and magnetoelectric coupling phenomena, they can be grouped into two fundamental categories: In the split-order-parameter multiferroics the electric and the magnetic order emerge independently - at separate transition temperatures and due to a different microscopic origin. Hexagonal (h-) $\mathrm{HoMnO}_{3}$ is a prominent example with $T_{\mathrm{C}}^{\mathrm{el}}=875 \mathrm{~K}$ and $T_{\mathrm{N}}^{\mathrm{mag}}=75 \mathrm{~K} \stackrel{3.4}{\text { In }}$ the joint-order-parameter multiferroics the electric and the magnetic order have a common microscopic origin. For instance, in $\mathrm{Ni}_{3} \mathrm{~V}_{2} \mathrm{O}_{8}$ the magnetic long-range order violates the spatial inversion symmetry and induces a spontaneous polarization via the antisymmetric Dzyaloshinskii-Moriya interaction. $\frac{5}{5}$ Consequently, the symmetry-breaking magnetic and electric order parameters arise at the same temperature $(T=6.3 \mathrm{~K})$.

An universal property of all multiferroics is a substantial reduction of the number of symmetry elements by the multiple order which implies the formation of a large number of domains. In particular, incommensurate systems have a lower symmetry compared to the non-ferroic state because of the loss of the three-dimensional translation symmetry. With the unusual nature of the magnetic and electric order in multiferroics and the additional degree of freedom arising from their interaction, novel types of domains and domain-related effects are expected. Unfortunately, we still have a very fragmentary understanding of the complex domain states in multiferroics - the diversity of multiferroic domains is largely ignored, although domains are the key to many macroscopic properties of technological relevance such as coercive field, electrical resistivity, and switching dynamics.

Investigations of domains in multiferroics so far are focussed on orientation domains, i.e., domains differing in the orientation of magnetic and/or electric order parameters ${ }^{6-9}$ In contrast, investigations of translation domains, i.e., domains differing in the phase but not the orientation of the order parameter (so that they are also called anti-phase domains), are extremely rare because their experimental observation is a very challenging task and their technological feasibility is questionable $\stackrel{10}{0}$ However, the substantial role the walls between translation domains can play for magnetoelectric interactions was pointed out only recently $\underline{11,12}$

In this paper we consider two complementary cases of translation domains in multiferroics that demonstrate the extraordinary scope of manifestations: a split-order-parameter multiferroic with commensurate order and a joint-orderparameter multiferroic with incommensurate order. Here, h$\mathrm{YMnO}_{3}$ and $\mathrm{MnWO}_{4}$ were chosen as model compounds because their magnetic and electric properties are well understood. This is a good basis for studying the formation and properties of their translation domains which are visualized using piezo-response force microscopy (PFM) and optical second harmonic generation (SHG). We discuss the associated symmetry-breaking order parameters and compare spatially resolved measurements of the domain topology in the two compounds. Our analysis shows that they can be regarded as end cases in the classification of translation domains in multiferroics. In $\mathrm{h}-\mathrm{YMnO}_{3}$ translation domains emerge due to discrete symmetry violations by a commensurate order parameter strongly coupling to the crystal lattice. In $\mathrm{MnWO}_{4}$ translation domains emerge due to continuous symmetry violations by an incommensurate order parameter. Here, the translation domains are decoupled from the crystallographic structure.

\section{ORDER PARAMETERS}

\section{A. Commensurate h- $\mathrm{YMnO}_{3}$}

In $\mathrm{h}-\mathrm{YMnO}_{3}$ translation domains appear in conjunction with the ferroelectric order which emerges in two steps:13,14 A tripling of the paraelectric unit cell at $1350 \mathrm{~K}$ and emergence of a spontaneous polarization $\pm P_{z}$ along the hexagonal axis at $1100 \mathrm{~K} \stackrel{15}{ }$ The reduction of the space symmetry by the two 


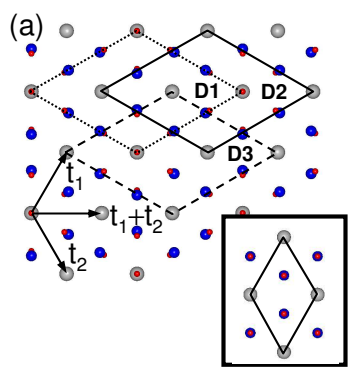

$\mathrm{t}_{1} \rightarrow \phi_{1}=2 \pi / 3$

$\mathrm{t}_{2} \rightarrow \phi_{2}=4 \pi / 3$

$\mathrm{t}_{1}+\mathrm{t}_{2} \rightarrow \phi_{3}=0,2 \pi$

(b)

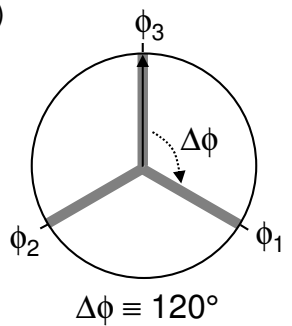

(c)

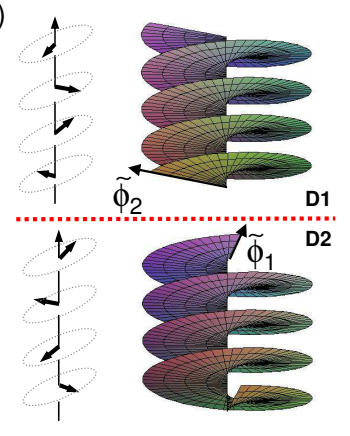

$\Delta \widetilde{\phi}=\widetilde{\phi}_{2}-\widetilde{\phi}_{1}$

(d)

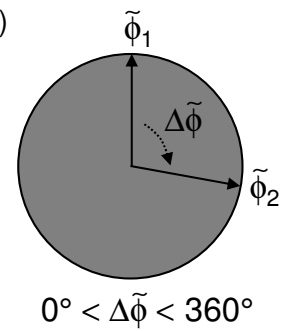

FIG. 1: Origin of translation domains in $\mathrm{h}-\mathrm{YMnO}_{3}$ and $\mathrm{MnWO}_{4}$. (a) Schematic illustration of the three possible ferroelectric unit cells in $\mathrm{h}-\mathrm{YMnO}_{3}$ and the translations $t_{1}, t_{2}$, and $t_{1}+t_{2}$ lost as symmetry operations with respect to the paraelectric unit cell (inset). (b) Polar plot of the order-parameter space of the ferroelectric phase of $\mathrm{h}-\mathrm{YMnO}_{3}$. The translations lost as symmetry operations have a discrete image in the order-parameter space so that each translation domain can be identified by a specific phase $\phi_{j}(j=1,2,3)$. (c) Schematic illustration of the elliptical spin spiral in the multiferroic phase of $\mathrm{MnWO}_{4}$ (left) and its dense continuation (right). (d) Polar plot of the order-parameter phase in the multiferroic state of $\mathrm{MnWO}_{4}$. Here, the translations lost as symmetry operations have a dense image in the order-parameter space. Consequently, $\tilde{\phi}_{j}$ can have any value $0^{\circ}<\tilde{\phi}_{j}<360^{\circ}$.

transitions is $P 6_{3} / \mathrm{mmc} \rightarrow P 6_{3} \mathrm{~cm}$.

Because of the enlargement of the unit cell, which is sketched in Fig. 1(a), the translation symmetries $t_{1}, t_{2}$, and $t_{3}:=t_{1}+t_{2}$ of the high-temperature $P 6_{3} / m m c$ phase are violated. As a consequence, three different translation or trimerization domains denoted by $\alpha, \beta$, and $\gamma$ arise,,$\underline{11}$ each being represented by one of the three structurally nonequivalent unit cells depicted in Fig. 11a). The associated two-dimensional order parameters are

$$
\eta_{j}=\left(\begin{array}{c}
\delta \mathrm{e}^{i \phi_{j}} \\
\delta \mathrm{e}^{-i \phi_{j}}
\end{array}\right)(j=1,2,3)
$$

with $\mathbf{k}=\left(\frac{1}{3}, \frac{1}{3}, 0\right)$ as commensurate wave vector describing the tripling. The translation domains can be identified by their relative phase $\phi_{j}=\mathbf{k} \mathbf{t}_{j}$ with $\phi_{1}(\alpha)=\frac{2 \pi}{3}, \phi_{2}(\beta)=\frac{4 \pi}{3}$, and $\phi_{3}(\gamma)=0,2 \pi$. The values for $\phi_{j}$ are illustrated in the polar plot in Fig. 1(b). Their discreteness nicely reflects the commensurability of the translation domains in $\mathrm{h}-\mathrm{YMnO}_{3}$. Apparently, all the translation domain walls in this compound correspond to a discontinuity in the phase of the commensurate order pa-

rameter by $\left|\Delta \phi_{j \neq j^{\prime}}\right|=\left|\phi_{j}-\phi_{j^{\prime}}\right| \equiv 120^{\circ}$ with $j$ and $j^{\prime}$ denoting different domains.

\section{B. Incommensurate $\mathrm{MnWO}_{4}$}

As illustrated by Figs. 11 c) and (d) the situation in $\mathrm{MnWO}_{4}$ is quite different. Here, translation domains evolve due to incommensurate magnetic long-range order ${ }^{10,16,17}$ First, a sinusoidal spin-density wave with the propagation vector $\mathbf{k}=$ $\left(-0.214, \frac{1}{2}, 0.457\right)$ emerges at $13.5 \mathrm{~K}$. It is associated to a single two-dimensional order parameter $\zeta_{j} \underline{18}$ At $12.7 \mathrm{~K}$ the spin density wave turns into an elliptical spin spiral with the same propagation vector as before. The transition is driven by a second two-dimensional magnetic order parameter

$$
\xi_{j}=\left(\begin{array}{c}
\rho \mathrm{e}^{i \tilde{\phi}_{j}} \\
\rho \mathrm{e}^{-i \tilde{\phi}_{j}}
\end{array}\right)(j \in \mathbb{N}) .
$$

Along with this, a magnetically induced polarization $P_{y}$ along the $y$ axis arises. Thus, the system becomes multiferroic and orientation domains with $\pm P_{y}$ are formed. Note that it is the coexistence of the order parameters $\zeta_{j}$ and $\xi_{j}$, not the presence of $\xi_{j}$ by itself, that breaks the inversion symmetry and induces a spontaneous polarization and, thus, multiferroicity. In addition, $\zeta_{j}$ and $\xi_{j}$ give rise to translation domains that are associated to the incommensurate spin arrangement in Figs.1 (c). Figure1 (c) shows the elliptical spin spiral of the multiferroic phase in $\mathrm{MnWO}_{4}$ and its dense continuation. The incommensurate magnetic structure described by $\mathbf{k}$ causes a unit cell doubling along the $y$ axis and violates any translation symmetry in the $x z$ plane $\stackrel{17}{=}$ In contrast to commensurate $\mathrm{h}-\mathrm{YMnO}_{3}$, the translation symmetries violated by the incommensurate structure in $\mathrm{MnWO}_{4}$ have a dense image in the order-parameter space, see Figs.1(b) and11d). This gives rise to an infinite number of translation domains enumerated by

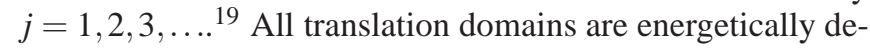
generated, because the free energy is invariant under the continuous rotation of the phase mode (phason) associated with the incommensurate spin spiral. Note that the phase between $\zeta_{j}$ and $\xi_{j}$ is unique so that it is sufficient to consider the order parameter $\xi_{j}$ only for distinguishing and enumerating the translation domains.

In an intuitive interpretation one can say that the phase difference $\Delta \tilde{\phi}_{j \neq j^{\prime}}=\tilde{\phi}_{j}-\tilde{\phi}_{j^{\prime}}$ appearing at a domain wall corresponds to a discontinuity in the spatial precession of the incommensurate spin spiral. Any value $\Delta \tilde{\phi}_{j \neq j^{\prime}} \in\left[0^{\circ}, 360^{\circ}\right]$ is possible due to the incommensurate nature of the magnetic order. Note that the identification of a translation domain is ambiguous. The phase $\tilde{\phi}$ changes continuously across a domain so that there is no absolute criterion for labelling it. Only the phase difference occurring at its boundary defines a domain wall and, thus, distinguishes different domains.

In summary, two fundamentally different types of translation domains are present in $\mathrm{h}-\mathrm{YMnO}_{3}$ and $\mathrm{MnWO}_{4}$ : In h$\mathrm{YMnO}_{3}$ a finite set of translation domains is generated by the commensurate order parameter and a universal phase shift 
of $120^{\circ}$ distinguishes different domains. The domains represent a pronounced structural discontinuity and are therefore expected to interact strongly with the underlying crystal lattice and the ferroelectric displacement. In contrast, $\mathrm{MnWO}_{4}$ can develop an infinite number of translation domains caused by the continuous symmetry breaking of the associated incommensurate magnetic order parameter so that the phase shift at the domain wall can have any value $0^{\circ}<\Delta \tilde{\phi}<360^{\circ}$. Due to this continuous nature only a weak coupling between the translation domains and the crystal lattice is expected.

\section{DOMAIN TOPOLOGY}

\section{A. Commensurate h-YMnO}

For investigating the translation domains in h- $\mathrm{YMnO}_{3}$, flux-grown $z$-oriented platelets with a lateral extension of a few millimeters and a thickness in the order of $100 \mu \mathrm{m}$ were used. After chemical-mechanical polishing with a silica slurry the distribution of domains was measured by PFM.

Figure 2(a) shows the $x y$ plane of h- $\mathrm{YMnO}_{3}$ under ambient conditions. The PFM image reveals domains of $\lesssim 1 \mu \mathrm{m}$ with two grey levels corresponding to ferroelectric domains with the polarization pointing parallel $+P_{z}$ (bright) or antiparallel $-P_{z}$ (dark) to the $z$ axis. The distribution of the domains is striking. All across the sample, a domain structure with meeting points of six domains is obtained. No exceptions are detected - whenever the ferroelectric domains approach one another the characteristic kaleidoscopic pattern is formed. The resulting topology is almost isotropic and can be observed in all three spatial dimensions ${ }^{20}$

The kaleidoscopic pattern with its meeting points of six domains can be understood on the basis of Figs. 1 (a) and 11(b): In the ferroelectric phase each of the three possible translation domains $\alpha, \beta$, and $\gamma$ can exhibit either a positive or a negative spontaneous polarization, so that six different trimerization-polarization domains are expected. As shown in Ref. 11 meetings of different translation domains require a reversal of polarization in order to be stable. Therefore, when the three possible translation domains meet in one point, this can only be solved by an arrangement of all six translationpolarization domains. In Ref. 20 it was shown that the arrangement has to be a sequence of the type shown in Fig.2(d) where the two order parameters $\eta$ and $P_{z}$ of h- $\mathrm{YMnO}_{3}$ change simultaneously at each domain wall. For instance, this allows an $\alpha^{+}, \beta^{-}, \gamma^{+}, \alpha^{-}, \beta^{+}, \gamma^{-}$sequence of domains around the meeting point, but it forbids an $\alpha^{+}, \alpha^{-}, \beta^{+}, \beta^{-}, \gamma^{+}, \gamma^{-}$arrangement. As explained in Ref. 20 the pairing of orientation and translation domain walls is due to the electrostatic discontinuity occurring at the translation domain walls. Hence, h$\mathrm{YMnO}_{3}$ is a remarkable example where translation domains, in contrast to the common belief, manifest in a very pronounced way. In fact, the translation domains control the distribution of the orientation domains in $\mathrm{h}-\mathrm{YMnO}_{3}$ and with this the distribution of the ferroelectric polarization. The electrostatic mechanism behind this reflects the electrostatic, rather unusual nature of the ferroelectric order in $\mathrm{h}-\mathrm{YMnO}_{3}$ which,
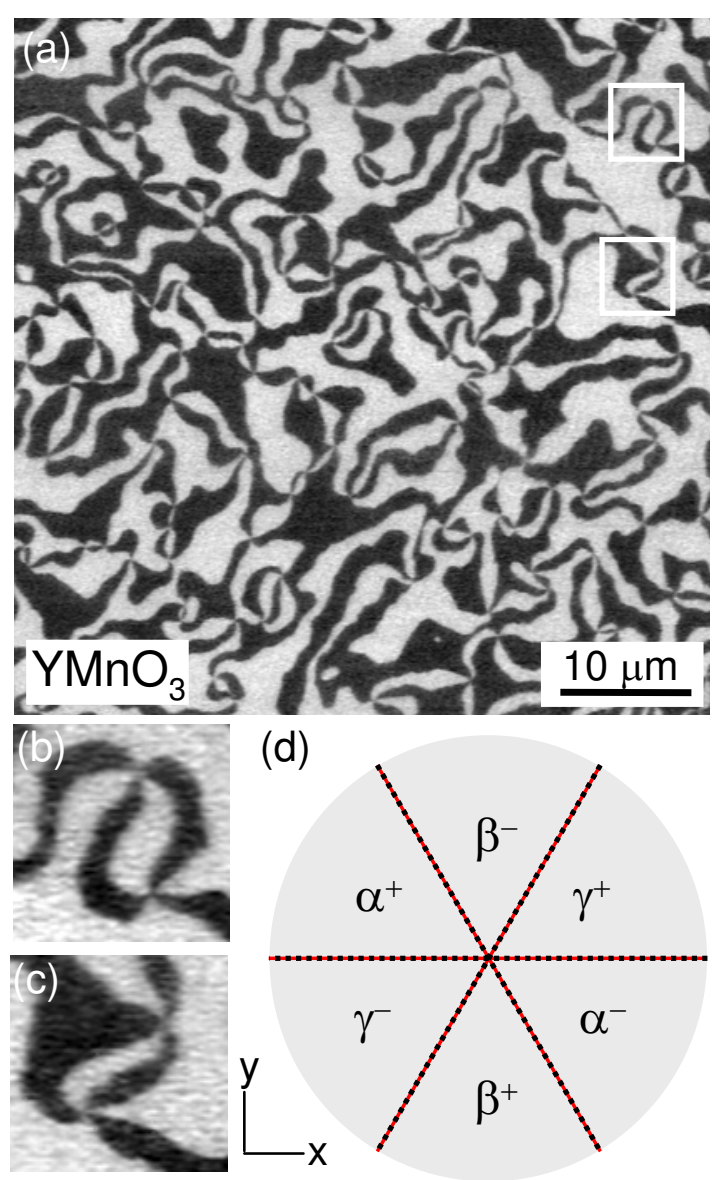

FIG. 2: (a) PFM image of the $x y$ plane of an as-grown h- $\mathrm{YMnO}_{3}$ crystal. Bright and dark areas correspond to ferroelectric domains with $+P_{z}$ and $-P_{z}$, respectively. (b) and (c) Enlargement of the domain intersections marked by boxes in panel (a). (d) Schematic of the intersections. Note that only one type of domain wall is present because of the rigid coupling of orientation and translation domains.

in turn, is common to split-order multiferroics.

\section{B. Incommensurate $\mathrm{MnWO}_{4}$}

We now turn to the complementary case, i.e., translation domains in an incommensurate joint-order-parameter multiferroic, namely $\mathrm{MnWO}_{4}$. The domain topology of $\mathrm{MnWO}_{4}$ was investigated by SHG since it is the only method allowing one to image antiferromagnetic translation domains, based on its coupling to the phase of the magnetic order parameter. ${ }^{21}$ SHG describes the induction of a dipole oscillation $\mathbf{P}(2 \omega)$ by an incident electromagnetic light wave given by $\mathbf{E}(\omega)$. It is described by the equation $P_{i}(2 \omega)=\varepsilon_{0} \chi_{i j k} E_{j}(\omega) E_{k}(\omega)$ with $\hat{\chi}$ as nonlinear susceptibility of the material in which the optical frequency doubling occurs. The set of components $\chi_{i j k} \neq 0$ reflects the symmetry and long-range order of the material. They can couple to the orientation and phase of the order parameter and thus be used to reveal the corresponding domain structure. In the following we will focus on the discussion of 

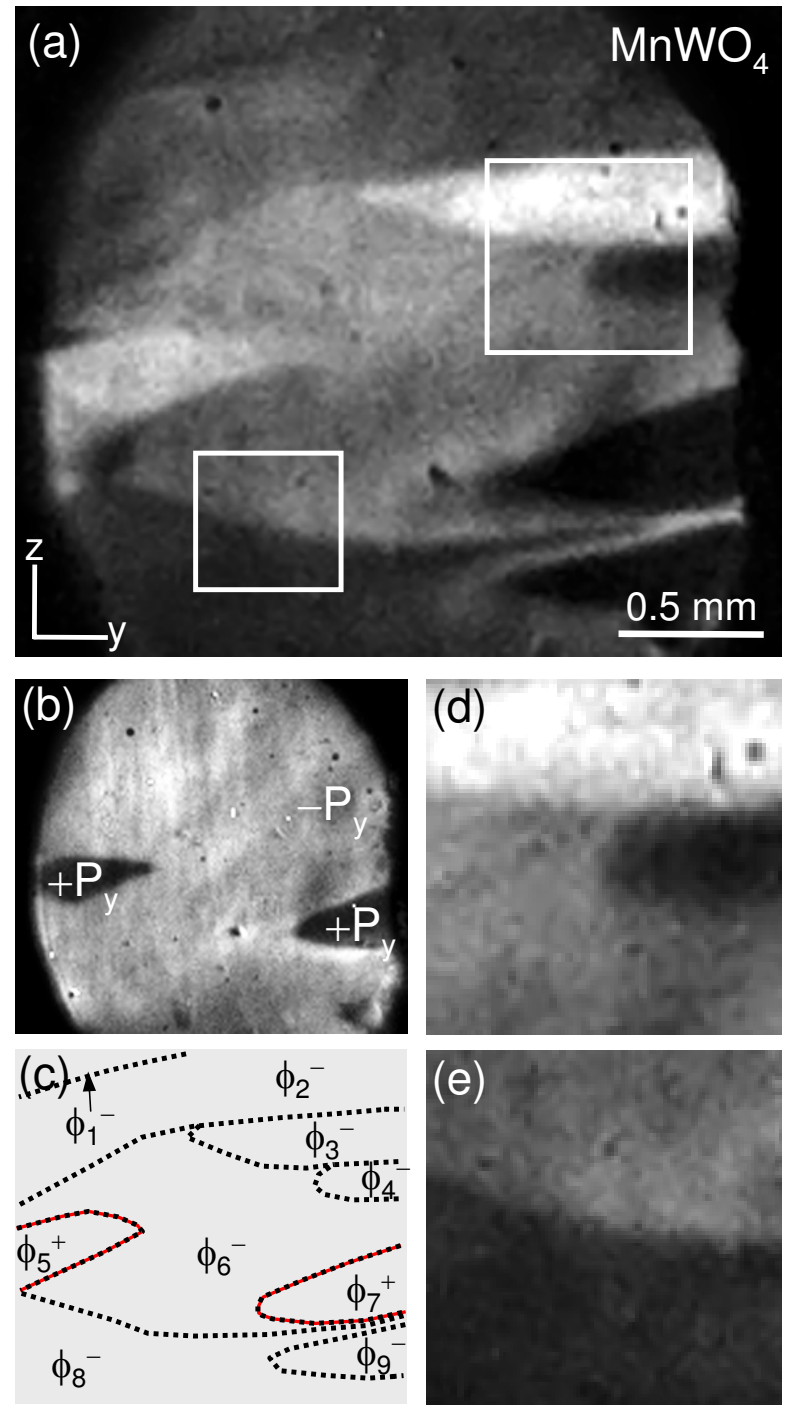

FIG. 3: (a) Coexistence of orientation and translation domains in the $y z$ plane of zero-field-cooled $\mathrm{MnWO}_{4}$. The SHG image was gained in the multiferroic phase at $8 \mathrm{~K}$ and shows a mixture of translation-polarization domain walls and translation-only domain walls. (b) Distribution of the ferroelectric orientation domains. (c) Schematic of the image in (a). Red solid lines denote the distribution of orientation domains of opposite polarization $\left(+P_{y}\right.$ or $\left.-P_{y}\right)$. Black dotted lines separate different translation domains labelled by $\phi_{j},(j=1-9)$. (d) and (e) Magnified meeting points of domains.

the domain pattern observed in $\mathrm{MnWO}_{4}$ whereas the discussion of the technical details of the SHG in $\mathrm{MnWO}_{4}$ are found elsewhere. 8,10,16,22

For the SHG measurements melt-grown $\mathrm{MnWO}_{4}$ samples were prepared in the same way as the $\mathrm{h}-\mathrm{YMnO}_{3}$ samples and measured in a transmission geometry. ${ }^{21,23}$ The samples had a lateral extension of $2.6 \times 3.2 \mathrm{~mm}$ and a thickness of $830 \mu \mathrm{m}$. Figure 3 displays a SHG image of the $y z$ plane exposed in the multiferroic phase at $8 \mathrm{~K}$. The image reveals differently shaded regions separated by abrupt changes of the SHG intensity. These regions correspond to different multiferroic do- mains. The different brightness is caused by the dependence of the amplitude and phase of the SHG contribution on the relative orientation and phase of the order parameters $\zeta$ and $\xi .21$ Thus, Fig. 3. (a) displays both orientation and translation domains. For disentangling them, the wavelength and polarization of the incident light in Fig. 3(b) were chosen such that only the ferroelectric orientation domains distinguished by their spontaneous polarization $\pm P_{y}$ were probed. This reveals two degrees of brightness corresponding to domains with $+P_{y}$ and $-P_{y}$. By comparing Figs. 3 a) and (b) two different types of domain walls in $\mathrm{MnWO}_{4}$ can be distinguished: (i) polarization-translation walls, and (ii) translation-only walls.

(i) At the domain walls marked by the red dotted lines in Fig. 3.c) the orientation of $P_{y}$ is reversed and a phase discontinuity of $\tilde{\phi}$ in the complex order parameter of Equation 2 occurs. The latter can be concluded because the change of brightness occurring upon crossing the polarization wall is not unique so that aside from the spontaneous polarization another aspect of the order must have changed - here the phase $\tilde{\phi}$ is the only option.

(ii) Figure 3 (a) reveals additional domain structures within the polarization domain denoted by $-P_{y}$. Here, the abrupt changes in the SHG yield correspond to translation-only walls at which only the phase $\tilde{\phi}$ of $\xi$ changes. The domains are distinguishable by SHG because of the aforementioned correspondence between the phase shifts of the SHG wave and the order parameter $\xi .10,22$ As sketched in Fig. 3 (c) more than six translation domains are resolved in Fig.3 (a). This observation is consistent with the effective continuous symmetry breaking by the associated incommensurate order parameter giving rise to an unlimited number of translation domains.

In order to point out peculiar features of the topology of the translation domains Figs. 3] d) and (e) show magnifications of regions revealing, respectively, a meeting point of three translation domains and a line separating two translation domains. The two SHG images indicate that the topology of the incommensurate translation domains is not subject to such rigid constraints as in the case of the commensurate splitorder-parameter multiferroic $\mathrm{h}-\mathrm{YMnO}_{3}$. In the joint-orderparameter multiferroic $\mathrm{MnWO}_{4}$ the number of translation domains that can meet in one point is not limited from the point of view of symmetry. The evolution of an arbitrary number of translation domains is a specific property of incommensurate systems, clearly separating them from commensurate matter, where a well-defined number of translation domains emerges in the ordered state. In addition, translation domains in incommensurate systems expand the established concept of translation domains by involving translations exceeding the expansion of the unit cell. Finally, as pointed out earlier, 19 incommensurabilities do not only increase the number of allowed domain states - they can also have a strong impact on the thermodynamic features related to the incommensurate phase transition. 


\section{CONCLUSION}

A violation of the three-dimensional translation symmetry by magnetic, electrical, or structural degrees of freedom gives rise to different types of translation in multiferroics. In contrast to orientation domains with a unique orientation of the corresponding order parameter the translation domains were largely ignored in literature so far because of their "hidden" nature. Here we demonstrated two end cases on the range of translation domains: commensurate ferroelectric translation domains in the split-order-parameter multiferroic $\mathrm{h}-\mathrm{YMnO}_{3}$ and incommensurate magnetic translation domains in the joint-order-parameter multiferroic $\mathrm{MnWO}_{4}$. The type of translation domain and the type of multiferroicity in these two compounds are intricately related. However, two basic scenarios become obvious: Translation symmetries can either be broken by commensurate or incommensurate long-range order with fundamental consequences regarding the number of possible domain states and the formation of domain walls.

Our results show that in spite of their generally subtle nature, translation domains can be associated to pronounced structural discontinuities. Consequently, the formation of translation domains must not be neglected when discussing the physical properties of multiferroics or other materials allowing the formation of translation domains. Moreover, the low symmetry at the site of the domain walls can be the origin of new correlation effects. Here, the manipulation of translation domains may even provide a handle for controlling the functionality of materials.

Acknowledgments: This work was supported by the DFG through the SFB 608 and the Deutsche Telekom AG. The authors thank Pièrre Tolédano for many fruitful discussions.
1 S.-W. Cheong and M. Mostovoy, Multiferroics: a magnetic twist for ferroelectricity, Nature Mat. 6 (2007), pp. 13 - 20.

2 Y. Tokura, Multiferroics - toward strong coupling between magnetization and polarization in a solid, J. Magn. Magn. Mater. 310 (2007), pp. $1145-1150$.

3 B.B. van Aken, T.T.M. Palstra, A. Filippetti, and N.A. Spaldin, The origin of ferroelectricity in magnetoelectric $\mathrm{YMnO}_{3}$, Nature Mat. 3 (2004), pp. $164-170$.

4 Th. Lottermoser, Th. Lonkai, U. Amann, D. Hohlwein, J. Ihringer, and M. Fiebig, Magnetic phase control by an electric field, Nature 430 (2004), pp. 541 - 544.

5 G. Lawes et al., Magnetically driven ferroelectric order in $\mathrm{Ni}_{3} \mathrm{~V}_{2} \mathrm{O}_{8}$, Phys. Rev. Lett. 95 (2005), 087205.

6 M. Fiebig, Th. Lottermoser, D. Fröhlich, A.V. Goltsev, and R.V. Pisarev, Observation of coupled magnetic and electric domains, Nature 419 (2002), pp. 818 - 820.

7 T. Zhao et al., Electrical control of antiferromagnetic domains in multiferroic $\mathrm{BiFeO}_{3}$ films at room temperature, Nature Mat. 5 (2006), pp. $823-829$.

8 D. Meier, N. Leo, M. Maringer, Th. Lottermoser, P. Becker, L. Bohatý, and M. Fiebig, Topology and manipulation of multiferroic domains in $\mathrm{MnWO}_{4}$, Phys. Rev. B 80 (2009), 224420.

9 I. Cabrera et al., Coupled Magnetic and Ferroelectric Domains in Multiferroic $\mathrm{Ni}_{3} \mathrm{~V}_{2} \mathrm{O}_{8}$, Phys. Rev. Lett. 103 (2009), 087201.

10 D. Meier, N. Leo, Th. Lottermoser, P. Becker, L. Bohatý, and M. Fiebig, Imaging of hybrid-multiferroic and translation domains in a spin-spiral ferroelectric, Mater. Res. Soc. Symp. Proc. 1199 (2010), 1199-F02-07.

11 T. Choi, Y. Horibe, H.T. Yi, Y.J. Choi, W. Wu, and S.-W. Cheong, Insulating interlocked ferroelectric and structural antiphase domain walls in multiferroic $\mathrm{YMnO}_{3}$, Nature Mat. 9 (2010), pp. 253 $-257$.

12 M. Mostovoy, A whirlwind of opportunitues, Nature Mat. 9 (2010), pp. 188 - 190.

13 Th. Lonkai, D.G. Tomuta, U. Amann, J. Ihringer, R.W.A. Hendrikx, D.M. Többens, and J.A. Mydosh, Development of the high- temperature phase of hexagonal manganites, Phys. Rev. B 69 (2004), 134108.

14 C.J. Fennie and K.M. Rabe, Ferroelectric transition in $\mathrm{YMnO}_{3}$ from first principles, Phys. Rev. B 72 (2005), 100103(R).

15 G. Nénert, M. Pollet, S. Marinel, G.R. Blake, A. Meetsma, and T.T.M. Palstra, Experimental evidence for an intermediate phase in the multiferroic $\mathrm{YMnO}_{3}$, J. Phys.: Condens. Matter 19 (2007), 466212.

16 D. Meier, M. Maringer, Th. Lottermoser, P. Becker, L. Bohatý, and M. Fiebig, Observation and coupling of domains in a spinspiral multiferroic, Phys. Rev. Lett. 102 (2009), 107202.

17 G. Lautenschläger, H. Weitzel, T. Vogt, R. Hock, A. Böhm, M. Bonnet, and H. Fuess, Magnetic phase transitions of $\mathrm{MnWO}_{4}$ studied by the use of neutron diffraction, Phys. Rev. B 48 (1993), pp. $6087-6098$.

18 P. Tolédano, B. Mettout, W. Schranz, and G. Krexner, Directional magnetoelectric effects in $\mathrm{MnWO}_{4}$ : magnetic sources of the electric polarization, J. Phys.: Condens. Matter 22 (2010), 065901.

19 B. Mettout, P. Tolédano, and M. Fiebig, Symmetry replication and toroidic effects in the multiferroic pyroxene $\mathrm{NaFeSi}_{2} \mathrm{O}_{6}$, Phys. Rev. B 81 (2010), 214417.

20 T. Jungk, Á. Hoffmann, M. Fiebig, and E. Soergel, Electrostatic topology of ferroelectric domains in $\mathrm{YMnO}_{3}$, Appl. Phys. Lett. 97 (2010), 012904.

21 M. Fiebig, V.V. Pavlov, and R.V. Pisarev, Second-harmonic generation as a tool for studying electronic and magnetic strucutres of crystals, J. Opt. Soc. Am. B 22 (2005), pp. $96-118$.

22 D. Meier, N. Leo, G. Yuan, Th. Lottermoser, P. Becker, L. Bohatý, and M. Fiebig, Second harmonic generation on incommensurate structures: The case of $\mathrm{MnWO}_{4}$, preprint: arXiv:1008.2864 (2010).

23 P. Becker, L. Bohatý, H. J. Eichler, H. Rhee, and A. A. Kaminskii, High-gain raman induced multiple stokes and anti-stokes generation in monoclinic multiferroic $\mathrm{MnWO}_{4}$ single crystals, Laser Phys. Lett. 4 (2007), pp. $884-889$. 